\title{
Septicemic Melioidosis in a Diabetic Patient from a Tertiary Care Centre in Kerala
}

Netto Jacob*

Department of Microbiology, Government Medical College, Kottayam, Kerala, India

"Corresponding author: Netto Jacob, Department of Microbiology, Government Medical College, Kottayam, Kerala, India, Tel: +919400475381; E-mail: nettomuthanattu@gmail.com

Received Date: Sep 12, 2018; Accepted Date: Nov 06, 2018; Published Date: Nov 15, 2018

Copyright: (C) 2018 Jacob N. This is an open-access article distributed under the terms of the Creative Commons Attribution License, which permits unrestricted use, distribution, and reproduction in any medium, provided the original author and source are credited.

\begin{abstract}
Septicemic melioidosis is often a fatal disease despite treatment with antibiotics such as ceftazidime to which Burkholderia pseudomallei is sensitive in vitro. We report a case of $B$. pseudomallei causing sepsis in a diabetic patient. A 62 year old diabetic farmer, referred from a peripheral hospital with high grade fever and altered sensorium. Blood culture revealed B. pseudomallei. The patient was treated with meropenemand responded well to the treatment.
\end{abstract}

Keywords: Septicemic melioidosis; Diabetic patient; Kerala

\section{Introduction}

The pathologist Alfred Whitmore and his assistant C. S. Krishnaswami first described melioidosis as a "glanders-like" disease among morphia addicts in Rangoon, Burma, in 1911. This disease, now termed as melioidosis, was named from the Greek "melis" (distemper of asses) and "eidos" (resemblance) by Stanton and Fletcher [1].

Melioidosis has emerged as a disease of public health importance in Southeast Asia and northern Australia [2]. The severity of disease and risk of mortality is highest in persons with underlying chronic diseases such as diabetes mellitus and chronic renal failure [3]. The three main modes of acquisition of this agent are inhalation, ingestion, and inoculation [2]. Minor wounds and abrasions are common in farmers during the planting season, and inoculation through these wounds during their occupational exposure might be the common mode of spread. The organism has also been considered as a potential agent for biological warfare and biological terrorism [4]. Only few cases have been reported from India and it is assumed to be due to lack of adequate facilities, where identification is possible [1]. But sporadic exported cases from India indicate that the disease is endemic in this region [5]. Clinical presentations include the bacteremic form, with or without septic shock, and the non bacteremic form, including pneumonia, genitourinary and soft tissue infections, osteomyelitis, and others [2].

\section{Case Report}

A 62 year old diabetic farmer was admitted to our hospital in an altered sensorium with a 6 weeks history of fever. He was on oral hypoglycemics for the past 4 years. Physical examination revealed high grade fever. Blood test showed a total count of 12,000 which later elevated to 14,000 . Blood sugar was $255 \mathrm{mg} / \mathrm{dl}$ with normal renal function. Peripheral smear reported as neutrophilic leukocytosis with toxic granules. CSF study was done and was normal. MRI brain revealed a pituitary macroadenoma without any pressure effects. No intracranial bleeding was there. Patient was started on Inj. Ceftriaxone $2 \mathrm{~g}$ iv BD and Inj. Acyclovir $500 \mathrm{mg}$ iv Q8H.

Later, as patient complained of a vague pain in left hypochondrium, a USG abdomen was done which revealed ill-defined hypoechoic lesion involving splenic parenchyma, largest measuring $2.1 \times 1.3 \mathrm{~cm}$. As the patient's condition was bad, abscesses were not drained. Blood culture sent to microbiology lab yielded $B$. pseudomallei sensitive to Ceftazidime, Meropenem, Doxycycline and Co-Trimoxazole, resistant to Ciprofloxacin, Chloramphenicol and Amoxyclav. Based on antibiotic sensitivity, he was started on Inj. Meropenem $1 \mathrm{~g}$ iv Q8H. Patient responded well to intravenous Meropenem therapy.

\section{Discussion}

Burkholderia pseudomallei, the causative agent of melioidosis, is a gram-negative bacterium with rounded ends and bipolar staining, seen in tropical and subtropical soils and waters. It has got a high degree of virulence and strong antibiotic resistance [2,6]. A number of risk factors for developing melioidosis such as diabetes, thalassemia, renal disease, and occupational exposure to surface water have been defined. Patients with diabetes mellitus, in particular, have a high incidence $[1,7]$. A male preponderance is evident in all melioidosis case series published. This likely reflects the outdoor work and occupational exposure to soil and water. The incidence is high during rainy seasons as the bacteria move to the surface with the rising water table and multiplies in the surface water and wet soil [7]. Pneumonia is the most frequent presentation of melioidosis and is involved in approximately half of all cases [2]. But when presenting as acute septicemic melioidosis, the mortality is high, atleast $34 \%$ despite treatment with ceftazidime, the current treatment of choice [3]. Isolation of $B$. pseudomallei from the body fluids of patients still remains as the gold standard in the diagnosis [2]. B. pseudomallei is frequently resistant to many antibiotics including aminoglycosides and first- or secondgeneration cephalosporins. Since other third-generation cephalosporins such as ceftriaxone are less active, ceftazidime is the treatment of choice [8]. Current interventions are early intravenous administration of antibiotics, ceftazidime or carbapenems, for 10 to 14 
Citation: Jacob N (2018) Septicemic Melioidosis in a Diabetic Patient from a Tertiary Care Centre in Kerala. Med Rep Case Stud 3: 169. doi: $10.4172 / 2572-5130.1000169$

Page 2 of 2

days, followed by oral administration of co-trimoxazole or cotrimoxazole doxycycline for 12 to 20 weeks [6].

In this case, the patient might have infected as a result of occupational exposure, a minor trauma which went unnoticed or it could be an earlier exposure as the disease is notorious for latency and reactivation. The presence of diabetes mellitus as a risk factor is accountable for the development of septic shock. Early treatment is also essential in case of melioidosis. Patient in sepsis with abscesses in spleen or liver, if you ruled out routine causes like infective endocarditis or HIV related abscesses should be started on Ceftazidime $(50 \mathrm{mg} / \mathrm{kg}$, up to $2 \mathrm{~g}$ ) every 6 hour or Meropenem $(25 \mathrm{mg} / \mathrm{kg}$, up to 1 g) every 8 hour even before the isolation of organism in cultures.

\section{References}

1. Cheng AC, Currie BJ (2005) Melioidosis: Epidemiology, Pathophysiology and Management. Clin Microbiol Rev 18: 383-416.
2. Redondo MC, Gómez M, Landaeta ME, Ríos H, Khalil R, et al. (2011) Melioidosis presenting as sepsis syndrome: a case report. Int J Infect Dis Mar 15: e217-e218.

3. Inglis TJ, Golledge CL, Clair A, Harvey J (2001) Case report: recovery from persistent septicemic melioidosis. Am J Trop Med Hyg 5: 76-82.

4. Subbalaxmi MV, Chandra N, Rao MN, Vemu L, Raju YS (2011) Burkholderia pseudomallei: an uncommon cause of bacteraemic pneumonia in a diabetic. Indian J Chest Dis Allied Sci 53: 185-187.

5. Dance DA (1991) Melioidosis: the tip of the iceberg? Clin Microbiol Rev 4: 52-60.

6. Jin J-L, Ning Y-X (2014) Septicemic melioidosis: a case report and literature review. J Thorac Dis 6: E1-E4.

7. Ramamoorthia K, Saravua K, Mukhyopadhyaya C, Barakura AS (2013) Melioidosis: an underdiagnosed disease in India (epidemiology, clinical features, and outcomes). Asian Biomed 7: 249-256.

8. Lu P-L, Tseng S-H (2005) Fatal septicemic melioidosis in a young military person possibly co-infected with Leptospira interrogans and Orientia tsutsugamushi. Kaohsiung J Med Sci 21: 173-178. 